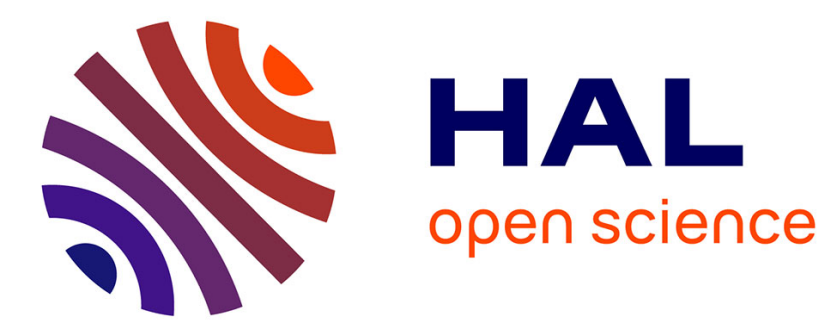

\title{
Chord length distribution in $d$-dimensional anisotropic Markov media
}

Coline Larmier, Alessandro Marinosci, Andrea Zoia

\section{To cite this version:}

Coline Larmier, Alessandro Marinosci, Andrea Zoia. Chord length distribution in $d$-dimensional anisotropic Markov media. Journal of Quantitative Spectroscopy and Radiative Transfer, 2018, 224, pp.403-414. 10.1016/j.jqsrt.2018.12.003 . cea-02339411

\section{HAL Id: cea-02339411 https://hal-cea.archives-ouvertes.fr/cea-02339411}

Submitted on 20 Nov 2019

HAL is a multi-disciplinary open access archive for the deposit and dissemination of scientific research documents, whether they are published or not. The documents may come from teaching and research institutions in France or abroad, or from public or private research centers.
L'archive ouverte pluridisciplinaire HAL, est destinée au dépôt et à la diffusion de documents scientifiques de niveau recherche, publiés ou non, émanant des établissements d'enseignement et de recherche français ou étrangers, des laboratoires publics ou privés. 


\title{
Chord length distribution in $d$-dimensional anisotropic Markov media
}

\author{
Coline Larmier ${ }^{\mathrm{a}}$, Alessandro Marinosci ${ }^{\mathrm{b}}$, Andrea Zoia ${ }^{\mathrm{a}, *}$ \\ ${ }^{a}$ DEN-Service d'études des réacteurs et de mathématiques appliquées (SERMA), CEA, Université Paris-Saclay, F-91191, Gif-sur-Yvette, France \\ ${ }^{b}$ Politecnico di Torino, Corso Duca degli Abruzzi 24, 10129, Torino, Italy.
}

\begin{abstract}
Markov media are often used as a prototype model in the analysis of linear particle transport in disordered materials. For this class of stochastic geometries, it is assumed that the chord lengths must follow an exponential distribution, with a direction-dependent average if anisotropy effects are to be taken into account. The practical realizability of Markov media in arbitrary dimension has been a long-standing open question. In this work we show that Poisson hyperplane tessellations provide an explicit construction for random media satisfying the Markov property and easily including anisotropy. The average chord length can be computed explicitly and is be shown to be intimately related to the statistical properties of the tessellation cells and in particular to their surface-to-volume ratio. A computer code that is able to generate anisotropic Poisson tessellations in arbitrary dimension restricted to a given finite domain is developed, and the convergence to exact asymptotic formulas for the chord length distribution and the polyhedral features of the tessellation cells is established by extensive Monte Carlo simulations in the limit of domains having an infinite size.
\end{abstract}

Keywords: Markov geometries, Poisson, Tessellations, Anisotropy, Chords, Monte Carlo

\section{Introduction}

Linear particle transport through random media is key to several applications in nuclear science (1), such as pebble-bed reactors (2), fluid-vapour mixtures in boiling water reactors (3), 5 radiation shielding by concrete structures (4), hydrodynamical instabilities in fusion pellets $(5$; 6, 7), the assessment of recriticality probability following core degradation (8; 9) and the impact of irregular aggregates in neutron absorbers (10) and MOX fuels (11). Moreover, random media concern also light propagation through turbid materials $(12 ; 14 ; 15)$ and tracer diffusion in biological tissues (16).

For the sake of clarity, and without loss of generality, in the following we will focus on binary stochastic mixing, where only two immiscible materials (say $\alpha$ and $\beta$ ) are randomly distributed according to some statistical law $(1 ; 17)$. For this class of problems, one is typically interested in obtaining the angular flux $\left\langle\varphi_{\alpha}(\mathbf{r}, \boldsymbol{\Omega})\right\rangle$ ensemble-averaged over those realizations that have material $\alpha$ at position $\mathbf{r}(17)$. It has been shown that $\left\langle\varphi_{\alpha}(\mathbf{r}, \mathbf{\Omega})\right\rangle$ is intimately related to the conditional probability $R_{\alpha}\left(\mathbf{r}^{\prime}, \mathbf{r}\right)$ that particle displacements from $\mathbf{r}^{\prime}$ to $\mathbf{r}$ fall within material $\alpha$, given that $\mathbf{r}$ is in material $\alpha(1,17)$. Assuming that the medium is homogeneous, all the spatial points have the same statistical properties, so that $R_{\alpha}\left(\mathbf{r}^{\prime}, \mathbf{r}\right)=R_{\alpha}(\ell, \mathbf{\Omega})$, where $\ell$ is the distance between $\mathbf{r}$ and $\mathbf{r}^{\prime}$ along the direction $\boldsymbol{\Omega}$. Reasonably, general results of broad applicability can only be obtained for statistical disorder models with spatial homogeneity. Under such hypothesis of invariance by translations, $R_{\alpha}(\ell, \mathbf{\Omega})$ repre- ${ }_{15}$ sents thus the probability that the material interface along the

\footnotetext{
${ }^{*}$ Corresponding author

Email address: andrea.zoia@cea.fr (Andrea Zoia)
}

direction $\boldsymbol{\Omega}$ from a given point within material $\alpha$ is larger than $\ell$, and can be expressed as

$$
R_{\alpha}(\ell, \mathbf{\Omega})=\frac{1}{\Lambda_{\alpha}(\mathbf{\Omega})} \int_{\ell}^{\infty} d \ell^{\prime} \int_{\ell^{\prime}}^{\infty} f_{\alpha}\left(\ell^{\prime \prime} \mid \mathbf{\Omega}\right) d \ell^{\prime \prime},
$$

where $f_{\alpha}(\ell \mid \boldsymbol{\Omega})$ is the probability density that the chord determined by intersecting an arbitrary line of orientation $\mathbf{\Omega}$ with the boundaries of the material chunk with label $\alpha$ has a length $\ell(18)$, and

$$
\Lambda_{\alpha}(\mathbf{\Omega})=\int_{0}^{\infty} \ell f_{\alpha}(\ell \mid \mathbf{\Omega}) d \ell
$$

is the average material chord length (i.e., the average linear material size) along direction $\boldsymbol{\Omega}$.

The chord length distribution $f_{\alpha}(\ell \mid \mathbf{\Omega})$, and the average material chord length $\Lambda_{\alpha}(\boldsymbol{\Omega})$ in particular, plays a prominent role in determining the behaviour of particle trajectories traversing the random medium $(1 ;, 19 ; 20)$. Assigning the chord length distribution $f_{\alpha}(\ell \mid \mathbf{\Omega})$ is tantamount to saying that the randomness of the medium is entirely described in terms of homogeneous renewal statistics, where the probability density that the distance to the interface from an arbitrary point within material $\alpha$ is $\ell$, namely,

$$
-\frac{\partial}{\partial \ell} R_{\alpha}(\ell, \mathbf{\Omega})=\frac{1}{\Lambda_{\alpha}(\mathbf{\Omega})} \int_{\ell}^{\infty} f_{\alpha}\left(\ell^{\prime} \mid \mathbf{\Omega}\right) d \ell^{\prime},
$$

only depends on the distance $\ell$ to the interface of material $\alpha$ along the direction $\boldsymbol{\Omega}(1 ; 17,21 ; 19,22)$.

The chord length distribution $f_{\alpha}(\ell \mid \boldsymbol{\Omega})$ has been thoroughly investigated for the case of random inclusions of hard spheres in a background matrix $(23,24 ; 25 ; 26,27 ; 28 ;, 29)$. For a comprehensive review, see, e.g., (30). In particular, it has been

July 21, 2018 
shown that for homogeneous and isotropic inclusions of poly- 60 dispersed hard spheres in dimension $d$ the average chord length within the background matrix is related to the random medium properties by

$$
\Lambda_{\alpha}=\frac{1-\vartheta}{\vartheta} \frac{\kappa_{d}}{\kappa_{d-1}} \frac{\left\langle r^{d}\right\rangle}{\left\langle r^{d-1}\right\rangle},
$$

where $\vartheta$ denotes the packing fraction (i.e., the volume fraction of the space filled by the spheres $), \kappa_{d}=\pi^{d / 2} / \Gamma(1+d / 2)$ is the volume of the unit sphere in dimension $d$, and $\left\langle r^{n}\right\rangle=\int r^{n} g(r) d r$ is the $n$-th moment of the sphere radius, $g(r)$ being the radius 20 distribution (24, 25). Moreover, accurate Monte Carlo simulations have shown that the associated chord length distribution $f_{\alpha}(\ell)$ is nearly exponential, with average provided by Eq. (4), 70 and that the accuracy of this approximation improves for increasingly diluted spheres, i.e., small $\vartheta(26,28)$, as expected on 25 theoretical grounds $(30)$.

The class of homogeneous Markov media, which is particularly relevant for applications $(1,4,5,6,14,15)$, assumes that 75 the chord length distribution is exactly exponential, namely,

$$
f_{\alpha}(\ell \mid \mathbf{\Omega})=\frac{1}{\Lambda_{\alpha}(\mathbf{\Omega})} \exp \left(-\frac{\ell}{\Lambda_{\alpha}(\mathbf{\Omega})}\right) .
$$

In this case, the probability per unit length to cross the interface of material $\alpha$ in direction $\boldsymbol{\Omega}$ has a Poisson distribution with parameter $1 / \Lambda_{\alpha}(\mathbf{\Omega})(1)$. As such, Markov mixing represents an idealized mathematical description for disordered media, de30 manding minimal information content $(1,30)$. Models satisfying homogeneous Markov mixing were introduced by Pomraning and co-workers for one-dimensional geometries of the rod or slab type, based on a Poisson point process on the line (1, 4). Extensions to two-dimensional flat or extruded isotropic con35 figurations have been later proposed (7, 31). The practical realizability of such Markov models having an exponential distribution $f_{\alpha}(\ell \mid \mathbf{\Omega})$ simultaneously in all directions for arbitrary $d$-dimensional geometry has been an open question for many years (4). In a recent work, we have shown that a stochastic 40 geometry model based on $d$-dimensional isotropic Poisson tessellations (i.e., random partitions by $(d-1)$-dimensional hyperplanes, for which explicit construction methods exist) induce homogeneous Markov mixing satisfying the exponential chord length distribution with a direction-independent average

${ }_{45} \Lambda_{\alpha}$ (32). It has been suggested that such geometries might be the only hyperplane tessellations to satisfy these proper- 85 ties (33, 34).

In this paper we further extend our findings to the broader class of anisotropic Poisson tessellations, which can be of interest to describe the effects of material stratification and preferential orientations $(35 ; 36)$. For this purpose, we will first 90 provide the construction algorithm for stochastic geometries inducing an exponential chord length distribution with directiondependent average $\Lambda_{\alpha}(\boldsymbol{\Omega})$ and then illustrate how the average chord length $\Lambda_{\alpha}(\mathbf{\Omega})$ is related to the statistical features of the random medium and to the underlying anisotropy law. This pa- 95 per is organized as follows: in Sec. 2 we will briefly introduce the class of $d$-dimensional anisotropic Poisson stochastic tessellations and detail their main statistical properties. In Sec. 3 we will focus on the chord length distribution. Then, in Sec. 4 we will numerically verify the behaviour of three-dimensional anisotropic Poisson tessellations by Monte Carlo methods for different anisotropy laws. Conclusions will be finally drawn in Sec.5 Technical details will be provided in Appendix A, Appendix B and Appendix C.

\section{Anisotropic Poisson tessellations}

Consider a disjoint aggregate $\left\{X_{i}\right\}, i=1,2, \cdots$ of bounded domains in the euclidean space $\mathbb{R}^{d}$, with $X_{i} \cap X_{j}=\emptyset$ for $i \neq j$. The aggregate is space-filling in $\mathbb{R}^{d}$ if the union of the domains $X_{i}$ yields the entire space $\mathbb{R}^{d}$. When the bounded domains are convex and space-filling, the interfaces between domain pairs are hyperplanes, and the domains $X_{i}$ are convex polyhedra. In this case, the aggregate $\left\{X_{i}\right\}$ is a tessellation of $\mathbb{R}^{d}$, and the domains $X_{i}$ are the cells of the tessellation. Stochastic tessellations are a class of probabilistic models where a given $d$-dimensional domain $\mathcal{D}$ is decomposed into random convex polyhedral cells by generating a collection of $(d-1)$-dimensional random hyperplanes in $\mathbb{R}^{d}(37,18,30,38)$. Poisson geometries are a prominent example of stochastic tessellations where the partition is induced by hyperplanes drawn from an underlying Poisson process $(37 ;$; 18 ; 38).

\subsection{Construction of Poisson tessellations}

We will detail the algorithm for the construction of Poisson tessellations restricted to an arbitrary $d$-dimensional domain $\mathcal{D}$, based on Monte Carlo methods. Anisotropy can be rather straightforwardly taken into account by generalizing the methods proposed for isotropic Poisson tessellations (39, 40, 32). We start by sampling a random number $N$ of hyperplanes from a Poisson distribution of intensity $\alpha_{d} \rho R$, where $R$ is the radius of the $d$-sphere circumscribed to $\mathcal{D}$ and $\rho$ is the so-called density of the tessellation, carrying the units of an inverse length. The dimension-dependent constant $\alpha_{d}$ reads

$$
\alpha_{d}=\frac{d \kappa_{d}}{\kappa_{d-1}}=2 \sqrt{\pi} \frac{\Gamma\left(\frac{d+1}{2}\right)}{\Gamma\left(\frac{d}{2}\right)} .
$$

This normalization of the tessellation density is such that the average chord length induced by the tessellation (in a sense to be elucidated later) is $1 / \rho$. Then, we generate the hyperplanes $K$ that will cut the domain $\mathcal{D}$. We choose a parameter $r$ uniformly in the interval $[-R, R]$ and additionally sample a unit vector $\mathbf{n}$ from a density $H(\mathbf{n})$ with support in the half-surface $\Omega_{d}^{+}$of the unit $d$-sphere. Denoting by $\mathbf{M}$ the point such that $\mathbf{O M}=r \mathbf{n}$, the random plane $K$ will pass trough $\mathbf{M}$ and have normal vector $\mathbf{n}$ (for an illustration in $d=3$, see the scheme in Fig. 1). By construction, this hyperplane does intersect the circumscribed $d$-sphere of radius $R$ but not necessarily $\mathcal{D}$. The procedure is iterated until $N$ random hyperplanes have been generated, which yields homogeneous but (generally) non-isotropic Poisson tessellations (38). Anisotropy affects the tessellations through the distribution $H(\mathbf{n})$ : isotropic Poisson tessellations are obtained as a special case when setting $H(\mathbf{n})=U(\mathbf{n})$, i.e., the uniform 


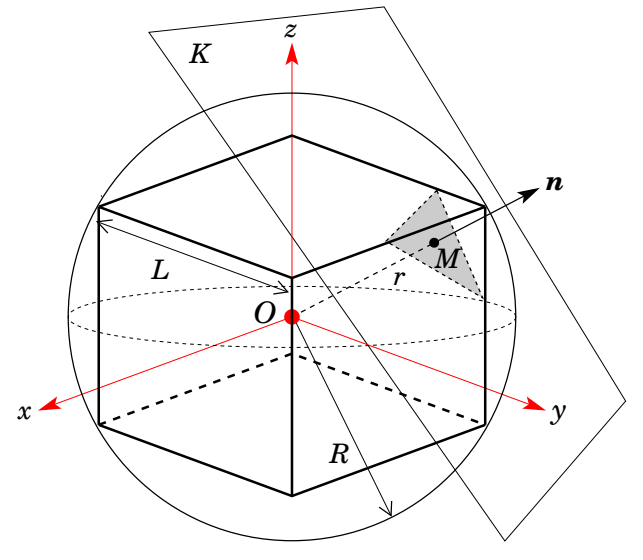

Figure 1: Cutting a cube in $\mathbb{R}^{3}$ with a random plane. A cube of side $L$ is centered in $O$. The circumscribed sphere centered in $O$ has a radius $R=\sqrt{3} L / 2$. The point $\mathbf{M}$ is defined by $\mathbf{M}=r \mathbf{n}$, where $r$ is uniformly sampled in the interval $[-R, R]$ and $\mathbf{n}$ is a random unit vector sampled from $H(\mathbf{n})$. The random plane $K$ of equation $n_{1} x+n_{2} y+n_{3} z=r$ is orthogonal to the vector $\mathbf{n}$ and intersects the point $\mathbf{M}$. The components of $\mathbf{n}$ can be expressed in spherical coordinates as: $n_{1}=\sin (\theta) \cos (\phi), n_{2}=\sin (\theta) \sin (\phi)$, and $n_{3}=\cos (\theta)$, where $\theta$ is the polar angle (projection onto the $z$ axis), and $\phi$ is the azimuthal angle (projection onto the $x-y$ plane).

angular distribution over $\Omega_{d}^{+}$(for further details on the isotropic case, see (32)). Due to geometrical reasons, it is only possible to include anisotropy effects for $d \geq 2$. A few examples of realizations corresponding to various angular laws $H(\mathbf{n})$ are illustrated $^{130}$ in Fig. 2. The density $H(\mathbf{n})$ might admit a finite mass concentrated on a given direction $\mathbf{n}_{0}$, or more generally on a collection of discrete directions $\mathbf{n}_{i}$, with Dirac delta distributions (41, 33).

\subsection{Statistical properties of polyhedral cells}

Knowledge of the statistical properties of the polyhedral cells of the stochastic tessellations can be helpful in understanding the behaviour of particle streaming through the geometry. In this framework, the quantities of interest are: the volume $V_{d}$ of a cell in dimension $d$, which characterizes the spatial scale of the random medium; the surface $S_{d}$ and the number of faces $C_{d}$ of a cell, which are related to the connectivity of the medium. The inradius $r_{\text {in, } d}$, i.e., the radius of the largest sphere contained 115 in a (convex) polyhedron, and the outradius $r_{\text {out }, d}$, i.e., the radius of the smallest sphere enclosing a (convex) polyhedron, can be also useful so as to characterize the shape of the cells.

Such quantities are random variables, whose exact distributions are in most cases unfortunately not known (38). Nevertheless, analytical expressions have been established for a few low-order moments of the observables, in the limit case of domains having an infinite extension (18, 38, 37). The case of isotropic Poisson tessellations was first addressed by the pioneering work of Goudsmit for $d=2(42)$ and later thoroughly explored by Miles first for $d=2$ and then for higher dimensions in a series of groundbreaking works (41; 37; 33). Not surprisingly, the derivation of exact results for anisotropic tessellations is even more demanding: the case $d=2$ was investigated by Miles (41), whereas formulas for higher dimensions (a)

(b)
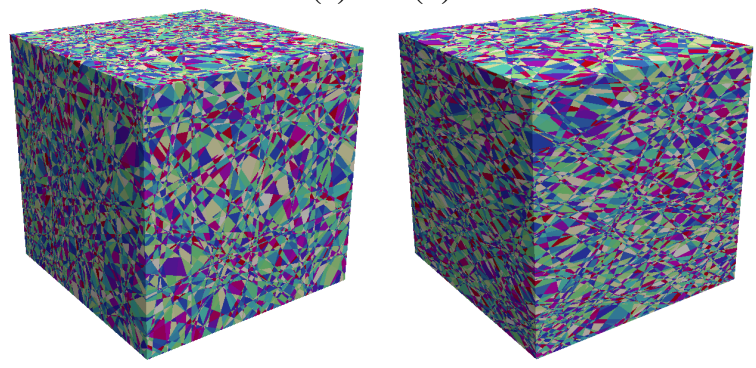

(c)

(d)
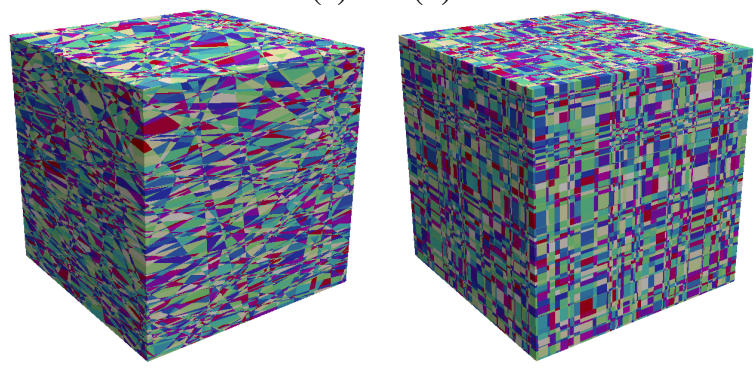

Figure 2: Realizations of three-dimensional anisotropic Poisson tessellations restricted to a box of side $L$, with various angular laws $H(\mathbf{n})=H(\theta, \phi)$. For all realizations, we have chosen $L=100$ and $\rho=1$. Case a) isotropic angular distribution ; b) quadratic angular distribution ; c) histogram distribution ; d) box distribution. The corresponding laws are detailed in $\mathrm{Sec} 4.1$

were found more recently and are still a subject of active research $(38,43,44)$.

As expected, for non-isotropic Poisson tessellations the obtained formulas generally depend on the angular distribution $H(\mathbf{n})$. The following results have been established for infinite domains (38). The first moment of the volume $V_{d}$ obeys

$$
\left\langle V_{d}\right\rangle=\frac{d !}{\zeta_{d}} \frac{1}{\rho^{d}},
$$

where the constant $\zeta_{q}$ depends on the angular distribution $H(\mathbf{n})$ and is defined as

$$
\zeta_{q}=\left(\frac{\alpha_{d}}{2}\right)^{q} \int_{\Omega_{d}^{+}} \cdots \int_{\Omega_{d}^{+}}\left[\mathbf{n}_{1}, \cdots, \mathbf{n}_{q}\right] d H\left(\mathbf{n}_{1}\right) \cdots d H\left(\mathbf{n}_{q}\right),
$$

$q \geq 1$, the integrals being extended over $\Omega_{d}^{+}$. Here the quantity $\left[\mathbf{n}_{1}, \cdots, \mathbf{n}_{q}\right]$ denotes the $q$-space determinant ${ }^{1}$ of the unit vectors $\mathbf{n}_{1}, \cdots, \mathbf{n}_{q}(38)$. As a particular case, for isotropic tessellations Eq. (8) gives

$$
\zeta_{d}^{\text {iso }}=\frac{d ! \kappa_{d}}{2^{d}},
$$

and we have

$$
\left\langle V_{d}\right\rangle^{\text {iso }}=\frac{1}{\kappa_{d}}\left(\frac{2}{\rho}\right)^{d} .
$$

It can be shown that for any sufficiently well-behaved $H(\mathbf{n})$ the quantity $\zeta_{d}$ satisfies $\zeta_{d} \leq \zeta_{d}^{\text {iso }}(\underline{38})$, which allows establishing

${ }^{1}$ For $q=2$, e.g., the $q$-space determinant yields the area of the parallelogram spanned by $\mathbf{n}_{1}$ and $\mathbf{n}_{2}$; for $q=3$, it yields the volume of the parallelepiped spanned by $\mathbf{n}_{1}, \mathbf{n}_{2}$ and $\mathbf{n}_{3}$. 
some general inequalities for the moments of the polyhedral quantities (see Appendix A). The second moment of the volume $V_{d}$ reads

$$
\left\langle V_{d}^{2}\right\rangle=\zeta_{d} \eta_{d} \kappa_{d}\left\langle V_{d}\right\rangle^{2}
$$

where the constant $\eta_{q}$ also depends on the angular distribution $H(\mathbf{n})$ and is defined as (38)

$$
\eta_{q}=\int_{\Omega_{d}^{+}} \xi^{-q}(\mathbf{\Omega}) d U(\mathbf{\Omega}),
$$

$q \geq 0$, with

$$
\xi(\mathbf{\Omega})=\frac{\alpha_{d}}{2} \int_{\Omega_{d}^{+}}|\mathbf{n} \cdot \mathbf{\Omega}| d H(\mathbf{n}) .
$$

Remark that $\eta_{0}=1$. For isotropic tessellations,

$$
\xi^{\text {iso }}=\frac{\alpha_{d}}{2} \int_{\Omega_{d}^{+}}|\mathbf{n} \cdot \mathbf{\Omega}| d U(\mathbf{n})=1,
$$

and Eq. (12) yields $\eta_{d}^{\text {iso }}=1$. We have thus

$$
\left\langle V_{d}^{2}\right\rangle^{\text {iso }}=d !\left(\frac{2}{\rho^{2}}\right)^{d} .
$$

The second moment allows computing the coefficient of variation, namely,

$$
\mathrm{cv}^{2}=\frac{\left\langle V_{d}^{2}\right\rangle-\left\langle V_{d}\right\rangle^{2}}{\left\langle V_{d}\right\rangle^{2}},
$$

which conveys information on the spatial shape of a typical ${ }^{140}$ cell (33). From Eqs. (11) and (7), we obtain

$$
\mathrm{cv}^{2}=\zeta_{d} \eta_{d} \kappa_{d}-1
$$

Furthermore, an elegant recursive formula is known relating the correlations between the surface $S_{d}$ and the volume $V_{d}$ to the higher moments of the volume, namely,

$$
m\left\langle V_{d}^{m-1} S_{d}\right\rangle=\alpha_{d} \rho\left\langle V_{d}^{m}\right\rangle,
$$

for $m \geq 1$ (33). Eq. (18) has been shown to hold for any $H(\mathbf{n})(38)$. In particular, for $m=1$ this yields the first moment of $S_{d}$, i.e,

$$
\left\langle S_{d}\right\rangle=\alpha_{d} \rho\left\langle V_{d}\right\rangle,
$$

and for $m=2$ the correlations

$$
\left\langle V_{d} S_{d}\right\rangle=\frac{\alpha_{d} \rho}{2}\left\langle V_{d}^{2}\right\rangle
$$

Thus, the effect of $H(\mathbf{n})$ is only indirectly conveyed on $\left\langle S_{d}\right\rangle$ and $\left\langle V_{d} S_{d}\right\rangle$ through the behaviour of $\left\langle V_{d}\right\rangle$ and $\left\langle V_{d}^{2}\right\rangle$, respectively. It is interesting to observe that, although the average volume and surface depend on the angular law $H(\mathbf{n})$ through the function $\zeta_{d}$, the surface-to-volume ratio of the cells does not, and we get

$$
\frac{\left\langle S_{d}\right\rangle}{\left\langle V_{d}\right\rangle}=\alpha_{d} \rho
$$

depending only on the density $\rho$ and on the constant $\alpha_{d}$.

For the number of faces $C_{d}$ we have the average

$$
\left\langle C_{d}\right\rangle=2 d,
$$

which is a purely combinatorial result, independent of $\rho$ and $H(\mathbf{n})$.

Finally, the inradius $r_{\text {in, } d}$ has an exponential distribution of parameter $\alpha_{d} \rho$, independent of $H(\mathbf{n})$, and we have in particular

$$
\left\langle r_{\mathrm{in}, d}\right\rangle=\frac{1}{\alpha_{d} \rho} .
$$

For the outradius $r_{\text {out }, d}$, the distribution is not known, and to the best of our knowledge the moments are not known, either, which prevents from using the theoretical ratio $\left\langle r_{\mathrm{in}, d}\right\rangle /\left\langle r_{\mathrm{out}, d}\right\rangle$ to extract information on the shape of the cells.

A special case of anisotropic Poisson tessellations corresponds to taking fixed orientations parallel to the orthogonal Cartesian axes in $\mathbb{R}^{d}$. In the simplest form, each direction is taken with equal probability, which yields a quasi-isotropic stochastic geometry: for these so-called Poisson-Box tessellations (41, 33), the functions $\zeta_{d}$ and $\eta_{d}$ can be computed exactly from Eqs. (8) and (12), respectively, and yield

$$
\zeta_{d}^{\mathrm{box}}=d !\left(\frac{\kappa_{d}}{2 \kappa_{d-1}}\right)^{d}
$$

and

$$
\eta_{d}^{\mathrm{box}}=\frac{1}{d ! \kappa_{d}}\left(\frac{4 \kappa_{d-1}}{\kappa_{d}}\right)^{d} .
$$

For isotropic Poisson tessellations, many other results are known for the low-order moments and the correlations of $V_{d}$, $S_{d}$ and $C_{d}$ (41; 33, 44), which will not be reported here. For an exhaustive review, the reader is referred to e.g., (38).

\section{Chord length distribution}

For anisotropic Poisson tessellations having density $\rho$ and infinite size, an arbitrary line with orientation $\mathbf{\Omega}$ will encounter a number of random hyperplanes per unit length distributed according to a Poisson distribution of density $\rho(\boldsymbol{\Omega})=\rho \xi(\boldsymbol{\Omega})$, depending on the anisotropy law $H(\mathbf{n})$ and on the direction $\boldsymbol{\Omega}(34)$. Conversely, the line will be cut by the hyperplanes into chords whose lengths $\ell$ obey the exponential distribution

$$
f(\ell \mid \mathbf{\Omega})=\rho(\mathbf{\Omega}) e^{-\ell \rho(\boldsymbol{\Omega})},
$$

depending on the fixed orientation $\mathbf{\Omega}$. Correspondingly, we have a direction-dependent average chord length

$$
\Lambda(\mathbf{\Omega})=\int \ell f(\ell \mid \mathbf{\Omega}) d \ell=\frac{1}{\rho(\mathbf{\Omega})} .
$$

The quantity $\Lambda(\boldsymbol{\Omega})$ physically represents the typical correlation length of the tessellation, i.e., the spatial scale of the local heterogeneity, in direction $\boldsymbol{\Omega}$ (1). For isotropic tessellations, from Eq. 14. we obtain in particular

$$
f^{\text {iso }}(\ell \mid \mathbf{\Omega})=f^{\text {iso }}(\ell)=\rho e^{-\ell \rho}
$$

for any orientation $\boldsymbol{\Omega}$. The average chord length reads then $\Lambda^{\text {iso }}(\boldsymbol{\Omega})=\Lambda^{\text {iso }}=1 / \rho$. 


\subsection{Colored Poisson tessellations}

In many applications, the cells of random tessellation are actually characterized by different physical properties, which can be formally described by assigning a distinct 'label' $i$ (also called 'color') to each cell, with a given probability $p_{i}$. Adjacent cells sharing the same label are merged, which gives rise to (generally) non-convex clusters, each composed of a random number of convex polyhedra.

The probability density $f_{i}(\ell \mid \mathbf{\Omega})$ of having a chord length $\ell$ though a cluster with label $i$ for lines with orientation $\boldsymbol{\Omega}$ can be obtained as follows. If $p_{i}$ is the probability for a cell to be assigned the label $i$, the probability that exactly $k$ adjacent cells share the same label ${ }^{2}$ is $P(k)=p_{i}^{k-1}\left(1-p_{i}\right)$. We can write then

$$
f_{i}(\ell \mid \mathbf{\Omega})=\sum_{k} f_{i}(\ell \mid \mathbf{\Omega} ; k) P(k),
$$

where $f_{i}(\ell \mid \Omega ; k)$ is the probability density of having a length $\ell$ if the cluster contains exactly $k$ cells in the direction $\boldsymbol{\Omega}$. Now, the length $\ell$ conditioned to $k$ cells is the sum of $k$ contributions $x_{j}(\mathbf{\Omega}), j=0, \cdots, k$, each obeying $f\left(x_{j} \mid \mathbf{\Omega}\right)$. Thus, $f_{i}(\ell \mid \mathbf{\Omega} ; k)$ is a Gamma distribution of parameters $\rho(\boldsymbol{\Omega})$ and $k$, namely,

$$
f_{i}(\ell \mid \mathbf{\Omega} ; k)=\frac{\rho^{k}(\mathbf{\Omega})}{\Gamma(k)} \ell^{k-1} e^{-\rho(\boldsymbol{\Omega}) \ell} .
$$

We have then

$$
f_{i}(\ell \mid \mathbf{\Omega})=\sum_{k} f_{i}(\ell \mid \mathbf{\Omega} ; k) P(k)=\rho_{i}(\mathbf{\Omega}) e^{-\rho_{i}(\mathbf{\Omega}) \ell},
$$

where $\rho_{i}(\mathbf{\Omega})=\rho(\mathbf{\Omega})\left(1-p_{i}\right)$. This means that anisotropic Poisson tessellations satisfy thus a strict Markov property: the chord length distribution for a cluster with label $i$ is exactly exponential, with rescaled parameter $\rho_{i}(\mathbf{\Omega})$. Finally, the average chord length through colored clusters reads

$$
\Lambda_{i}(\mathbf{\Omega})=\frac{1}{\rho_{i}(\mathbf{\Omega})}=\frac{\Lambda(\mathbf{\Omega})}{1-p_{i}},
$$

which relates the average material chord length $\Lambda_{i}(\boldsymbol{\Omega})$ to the correlation length $\Lambda(\boldsymbol{\Omega})$. Thus, the probability $p_{\alpha}$ of finding material $\alpha$ at any given spatial location is related to the average chord lengths by

$$
p_{\alpha}=\frac{\Lambda_{\alpha}(\mathbf{\Omega})}{\Lambda_{\alpha}(\mathbf{\Omega})+\Lambda_{\beta}(\mathbf{\Omega})},
$$

where the dependence on $\boldsymbol{\Omega}$ in $\Lambda_{i}(\boldsymbol{\Omega})$ cancels out to yield a direction-independent material probability $p_{\alpha}(1)$.

\subsection{Random line sections}

We will now examine the case where the chords are randomly distributed. Consider a disjoint aggregate of convex domains $\left\{X_{i}\right\} \subset X, i=1,2, \cdots, Q$, in $\mathbb{R}^{d}$ and a set $\mathcal{T}$ of uniformly and

\footnotetext{
${ }^{2}$ We are actually considering the probability conditioned to the first cell also sharing the same label.
}

isotropically distributed lines (for a precise definition, see Appendix B. From Eqs. (B.2) and (B.3), the average line section induced by $\mathcal{T}$ through the aggregate reads

$$
\mathbb{E}\left[\sum_{j=1}^{q} \ell_{j}\right]=\alpha_{d} \sum_{i=1}^{Q} \frac{V_{d}\left(X_{i}\right)}{S_{d}(X)}
$$

where $\ell_{j}, j=1, \cdots, q$, are the line sections of the non void intersections $\mathcal{T} \cap X_{j}$ between the lines and the domains composing the aggregate (46). Here $S_{d}(A)$ and $V_{d}(A)$ denote the surface and the volume of a domain $A$ in $\mathbb{R}^{d}$, respectively. The number $q$ of such intersections is itself random, with expected value

$$
\mathbb{E}[q]=\sum_{i=1}^{Q} \frac{S_{d}\left(X_{i}\right)}{S_{d}(X)} .
$$

By formally taking the ratio between Eqs. (34) and (35), the average line section for the domains composing the aggregate $\left\{X_{i}\right\}$ can be estimated by

$$
\lambda_{c} \equiv \frac{\mathbb{E}\left[\sum_{j=1}^{q} \ell_{j}\right]}{\mathbb{E}[q]}=\alpha_{d} \frac{\sum_{i=1}^{Q} V_{d}\left(X_{i}\right)}{\sum_{i=1}^{Q} S_{d}\left(X_{i}\right)} .
$$

Suppose now that the aggregate $\left\{X_{i}\right\} \subset X$ is a Poisson stochastic tessellation in $\mathbb{R}^{d}$. By taking ergodic averages over the constituents of the geometries $(46,34)$, in the limit of infinite size we obtain the average volume of a cell, namely,

$$
\lim _{Q \rightarrow \infty} \frac{1}{Q} \sum_{i=1}^{Q} V_{d}\left(X_{i}\right) \rightarrow\left\langle V_{d}\right\rangle,
$$

and the average surface of a cell

$$
\lim _{Q \rightarrow \infty} \frac{1}{Q} \sum_{i=1}^{Q} S_{d}\left(X_{i}\right) \rightarrow\left\langle S_{d}\right\rangle=\alpha_{d} \rho\left\langle V_{d}\right\rangle .
$$

Finally, combining Eqs. (36), 37) and (38), the average chord length $\Lambda_{c}$ through the cells of the tessellation induced by the random lines $\mathcal{T}$ can be estimated from (46)

$$
\Lambda_{c} \equiv \lim _{Q \rightarrow \infty} \lambda_{c} \rightarrow \alpha_{d} \frac{\left\langle V_{d}\right\rangle}{\left\langle S_{d}\right\rangle}=\frac{1}{\rho} .
$$

This remarkable result relates the chord length properties to the features of the tessellation cells, and shows that the average chord length $\Lambda_{c}$ induced by $\mathcal{T}$ through anisotropic Poisson tessellations of infinite size is simply equal to the inverse of the tessellation density $\rho$, In particular, $\Lambda_{c}$ is insensitive to the anisotropy law $H(\mathbf{n})$, although $V_{d}\left(X_{i}\right)$ and $S_{d}\left(X_{i}\right)$ separately depend on $H(\mathbf{n})$. Observe that

$$
\Lambda_{c} \leq \int_{\Omega_{d}^{+}} \Lambda(\mathbf{\Omega}) d U(\mathbf{\Omega})=\frac{\eta_{1}}{\rho},
$$

i.e., the average chord length $\Lambda_{c}$ cannot be obtained by averaging the direction-dependent correlation length $\Lambda(\mathbf{\Omega})$ over the uniform angular distribution. Rather, the definition in Eq. (39) formally corresponds to taking

$$
\frac{1}{\Lambda_{c}}=\int_{\Omega_{d}^{+}} \rho(\mathbf{\Omega}) d U(\mathbf{\Omega})=\rho,
$$


i.e., averaging the Poisson density $\rho(\boldsymbol{\Omega})=\rho \xi(\mathbf{\Omega})$ and then inverting the result. For isotropic tessellations, we simply have $\Lambda_{c}^{\text {iso }}=\Lambda^{\text {iso }}=1 / \rho$.

The result given in Eq. (4) for poly-dispersed spheres is actually closely related to the derivation of the average chord length as in Eq. (39). It suffices to observe (26, 30) that in this case the total volume of the background matrix is

$$
\left\langle V_{\text {matrix }}\right\rangle=(1-\vartheta) \kappa_{d}\left\langle r^{d}\right\rangle
$$

and that the surface of the interface between the spheres and the background matrix is

$$
\left\langle S_{\text {interface }}\right\rangle=\vartheta \Omega_{d}\left\langle r^{d-1}\right\rangle,
$$

where $\Omega_{d}=2 \pi^{d / 2} / \Gamma(d / 2)$ is the surface of the unit sphere in dimension $d$. Then, using $\Lambda_{c}=\alpha_{d}\left\langle V_{\text {matrix }}\right\rangle /\left\langle S_{\text {interface }}\right\rangle$ from Eq. (39) and $\Omega_{d}=d \kappa_{d}$, we are led to Eq. (4).

Additional information on the line sections in Markov media can be extracted for a special case of the higher-order moments. From Eqs. B.2 and (B.4), the $(d+1)$-th moment of the line section induced by $\mathcal{T}$ through an aggregate reads

$$
\mathbb{E}\left[\sum_{j=1}^{q} \ell_{j}^{d+1}\right]=\gamma_{d} \sum_{i=1}^{Q} \frac{V_{d}^{2}\left(X_{i}\right)}{S_{d}(X)}
$$

where $V_{d}^{2}(A)$ denote the square of the volume of a domain $A$ in $\mathbb{R}^{d}$ and the dimension-dependent constant $\gamma_{d}$ reads

$$
\gamma_{d}=\frac{d(d+1)}{\kappa_{d-1}}=\frac{2 d \Gamma\left(\frac{d+3}{2}\right)}{\pi^{(d-1) / 2}} .
$$

By formally taking the ratio between Eqs. 44) and (35), the $(d+1)$-th moment of the line section for the domains composing the aggregate $\left\{X_{i}\right\}$ can be estimated by

$$
\lambda_{c}^{d+1} \equiv \frac{\mathbb{E}\left[\sum_{j=1}^{q} \ell_{j}^{d+1}\right]}{\mathbb{E}[q]}=\gamma_{d} \frac{\sum_{i=1}^{Q} V_{d}^{2}\left(X_{i}\right)}{\sum_{i=1}^{Q} S_{d}\left(X_{i}\right)} .
$$

For Poisson stochastic tessellations, in the limit of infinite size ${ }^{185}$ we obtain

$$
\lim _{Q \rightarrow \infty} \frac{1}{Q} \sum_{i=1}^{Q} V_{d}^{2}\left(X_{i}\right) \rightarrow\left\langle V_{d}^{2}\right\rangle .
$$

Thus, combining Eqs. 46, 47) and 38, the $(d+1)$-th moment ${ }^{190}$ of the chord lengths through the cells of the tessellation induced by the random lines $\mathcal{T}$ can be estimated from (34)

$$
\Lambda_{c}^{d+1} \equiv \lim _{Q \rightarrow \infty} \lambda_{c}^{d+1} \rightarrow \gamma_{d} \frac{\left\langle V_{d}^{2}\right\rangle}{\left\langle S_{d}\right\rangle}=\eta_{d} \frac{\Gamma(d+2)}{\rho^{d+1}} .
$$

Similarly to Eq. (39), Eq. (48) relates the properties of the chord length to the features of the tessellation cells. To the best of our knowledge, no other expressions are known explicitly relating the moments of the chord lengths induced by the random lines $\mathcal{T}$ to the moments of the polyhedral features of the tessellation.200 It is interesting to remark that for lines thrown through the cells with arbitrary orientation $\mathbf{\Omega}$ we would have the $(d+1)$-th moment

$$
\Lambda^{d+1}(\mathbf{\Omega}) \equiv \int \ell^{d+1} f(\ell \mid \mathbf{\Omega}) d \ell=\frac{\Gamma(d+2)}{\rho^{d+1}(\mathbf{\Omega})} .
$$

In particular, for isotropic Poisson tessellations $\Lambda^{d+1 \text {,iso }}(\mathbf{\Omega})=$ $\Gamma(d+2) / \rho^{d+1}$, independent of the orientation $\boldsymbol{\Omega}$. In this case, $\Lambda_{c}^{d+1 \text {,iso }}=\Lambda^{d+1 \text {,iso }}$. The factor $\eta_{d}$ in Eq. (48) thus expresses the deviation with respect to the behaviour of isotropic tessellations having an exponential chord length distribution independent of $\mathbf{\Omega}$. Moreover, observe that we can explicitly compute the average of $\Lambda^{d+1}(\boldsymbol{\Omega})$ over the uniform distribution, which yields

$$
\Lambda_{c}^{d+1} \leq \int_{\Omega_{d}^{+}} \Lambda^{d+1}(\mathbf{\Omega}) d U(\mathbf{\Omega})=\eta_{d+1} \frac{\Gamma(d+2)}{\rho^{d+1}},
$$

similarly as what observed for the average chord length. Equality is attained for isotropic tessellations.

By comparing Eq. (39) to 48, we are led to the following picture: the average chord length $\Lambda_{c}$ is insensitive to the anisotropy law $H(\mathbf{n})$ of the tessellation, whereas the $(d+1)$-th moment $\Lambda_{c}^{d+1}$ generally does depend on $H(\mathbf{n})$. Since the properties of particle displacements through random media have been shown to be intimately related to the chord length distribution, and especially its lower-order moments, we therefore expect linear transport to be subtly affected by the effects of the anisotropy, which is coherent with the numerical findings that we have recently reported $(36)$.

\section{Monte Carlo analysis of anisotropic Poisson tessellations}

Anisotropic Poisson tessellations can be effectively used in order to derive reference solutions for particle transport in random media (36). For this purpose, we have implemented the construction algorithm provided above into a computer code that can generate an ensemble of realizations. Due to the complexity of the involved construction algorithms, for the case of isotropic tessellations careful verifications have been previously carried out by Monte Carlo simulation in order to verify that the generated tessellations obeyed the expected statistical properties (32). In this work we will extend our analysis to the case of anisotropic Poisson tessellations. The observables of interest, including the volume and surface of the cells, the number of faces per cell, and the chord lengths, are recorded for each realization: once a sufficiently large set has been generated, ensemble averages (and possibly also full distributions) are estimated. In the following, we illustrate some significant examples as a function of the angular law $H(\mathbf{n})$ in dimension $d=3$, which is relevant for physical applications.

\subsection{Choice of the angular distributions}

In (36) we have introduced a few laws $H(\mathbf{n})$, with $d H(\mathbf{n})=$ $d H(\theta, \phi)$ where the choice of the anisotropy law may mimic the impact of material stratification along the $z$ axis. For the sake of simplicity, we have assumed that the distributions $H(\mathbf{n})$ can be factorized with respect to the two variables, and that the distribution of $\phi$ is uniform (this ensures the invariance by rotation around the $z$ axis). In the following we briefly recall the 


\begin{tabular}{ccc}
\hline \hline Law $H(\mathbf{n})$ & $\zeta_{3}$ & $\eta_{3}$ \\
\hline Isotropic & $\pi \simeq 3.1416$ & 1 \\
Quadratic & $2.4232 \pm 2 \times 10^{-4}$ & $1.2796 \pm 6 \times 10^{-6}$ \\
Box & $16 / 9 \simeq 1.7777$ & $27 /(8 \pi) \simeq 1.0743$ \\
Histogram & $1.504 \pm 2 \times 10^{-4}$ & $2.0746 \pm 3 \times 10^{-6}$ \\
\hline \hline
\end{tabular}

Table 1: Numerical values for the constants $\zeta_{3}$ (see Eq. 8) and $\eta_{3}$ (see Eq. (12), for $d=3$ and various laws $H(\mathbf{n})$. Integrals have been computed by Monte Carlo sampling for quadratic and histogram laws (whence the error bars) and analytically for isotropic and box laws.

\begin{tabular}{ccc}
\hline \hline Law $H(\mathbf{n})$ & $\left\langle V_{3}\right\rangle$ & Monte Carlo \\
\hline Isotropic & $6 / \pi \simeq 1.90986$ & $1.907 \pm 4 \times 10^{-3}$ \\
Quadratic & $\simeq 2.4744$ & $2.459 \pm 5 \times 10^{-3}$ \\
Box & $27 / 8=3.375$ & $3.366 \pm 7 \times 10^{-3}$ \\
Histogram & $\simeq 3.987$ & $3.957 \pm 9 \times 10^{-3}$ \\
\hline \hline
\end{tabular}

Table 2: Average volume $\left\langle V_{3}\right\rangle$ of Poisson tessellations restricted to a box of side $L=200$, with various anisotropy laws $H(\mathbf{n})$, for $d=3$. The tessellation density is $\rho=1$ for all the angular laws $H(\mathbf{n})$. The limit value for infinite Poisson tessellations is given in Eq. (7).

definitions of the distributions that will be used for our Monte Carlo simulations.

We have considered a quadratic anisotropy

$$
H_{\text {quadratic }}(\mu, \phi)=\frac{3}{2 \pi} \mu^{2} \text { for }-1 \leq \mu<1,
$$

where $\mu=\cos (\theta)$, with $d H(\mathbf{n})=d H(\mu, \phi)$. This distribution has its minimum in $\mu=0$ and the maxima in $\mu= \pm 1$. Moreover, we have considered a piece-wise constant distribution

$$
H_{\text {histogram }}(\mu, \phi)=\frac{1}{A} \times \begin{cases}80 & \text { for }-1 \leq \mu<-0.95 \\ 4 & \text { for }-0.95 \leq \mu<-0.5 \\ 2 & \text { for }-0.5 \leq \mu<-0.25 \\ 1 & \text { for }-0.25 \leq \mu<0,\end{cases}
$$

and symmetric in the range $0<\mu<1$, which has maxima around $\mu= \pm 1$. The normalization constant reads $A=13.1 .^{230}$ The Poisson-Box tessellation with orientations parallel to the Cartesian axes reads

$$
\begin{gathered}
H_{\mathrm{box}}(\theta, \phi)=\frac{1}{3} \delta(\phi) \delta\left(\theta-\frac{\pi}{2}\right) \\
+\frac{1}{3} \delta\left(\phi-\frac{\pi}{2}\right) \delta\left(\theta-\frac{\pi}{2}\right)+\frac{1}{3} \delta(\theta) \frac{1}{\pi} .
\end{gathered}
$$

Finally, the isotropic distribution in $\Omega_{3}^{+}$yields

$$
H_{\text {iso }}(\theta, \phi)=\frac{1}{2 \pi} \sin (\theta)
$$

with $0 \leq \theta<\pi$ and $0 \leq \phi<\pi$. For the purpose of illustration, realizations of tessellations resulting from these distributions have been provided in Fig. 2 For the functional forms of the angular distributions $H(\mathbf{n})$ introduced here, the integrals 245 needed for the constants $\zeta_{3}$ (see Eq. (8)) and $\eta_{3}$ (see Eq. (12)) can be easily computed by Monte Carlo sampling, since they are both expressed as expected values over the angular distri-

\begin{tabular}{ccc}
\hline \hline Law $H(\mathbf{n})$ & $\left\langle V_{3}^{2}\right\rangle$ & Monte Carlo \\
\hline Isotropic & 48 & $47.8 \pm 0.2$ \\
Quadratic & $\simeq 79.6227$ & $79.2 \pm 0.3$ \\
Box & $729 / 8=91.125$ & $90.5 \pm 0.4$ \\
Histogram & $\simeq 207.9931$ & $206 \pm 1$ \\
\hline \hline
\end{tabular}

Table 3: Second moment of the volume $\left\langle V_{3}^{2}\right\rangle$ of Poisson tessellations restricted to a box of side $L=200$, with various anisotropy laws $H(\mathbf{n})$, for $d=3$. The tessellation density is $\rho=1$ for all the angular laws $H(\mathbf{n})$. The limit value for infinite Poisson tessellations is given in Eq. 11.

\begin{tabular}{ccc}
\hline \hline Law $H(\mathbf{n})$ & $\left\langle S_{3}\right\rangle$ & Monte Carlo \\
\hline Isotropic & $24 / \pi \simeq 7.63944$ & $7.63 \pm 0.01$ \\
Quadratic & $\simeq 9.986$ & $9.85 \pm 0.01$ \\
Box & $27 / 2=13.5$ & $13.48 \pm 0.02$ \\
Histogram & $\simeq 15.947$ & $15.84 \pm 0.03$ \\
\hline \hline
\end{tabular}

Table 4: Average surface $\left\langle S_{3}\right\rangle$ of Poisson tessellations restricted to a box of side $L=200$, with various anisotropy laws $H(\mathbf{n})$, for $d=3$. The tessellation density is $\rho=1$ for all the angular laws $H(\mathbf{n})$. The limit value for infinite Poisson tessellations is given in Eq. [19.

parallelepiped spanned by three unit vectors in the case of $\zeta_{3}$, and the scalar product of two unit vectors in the case of $\eta_{3}$, respectively. In special cases, such as for the isotropic and box distributions, the integrals can be computed analytically. The resulting constants $\zeta_{3}$ and $\eta_{3}$ are provided in Tab. 1. Finally, observe that we have $\alpha_{3}=4$ from Eq. (6) and $\gamma_{3}=12 / \pi$ from Eq. 45.

\subsection{Polyhedral features}

In order to verify the statistical features of the cells of the tessellations, we have generated anisotropic Poisson tessellations restricted to three-dimensional box of side $L$. For each angular law $H(\mathbf{n})$ described above, based on our computer code we have obtained a large number of realizations for increasing $L$ and fixed tessellation density $\rho=1$. The side of the box varies between $L=1$ and $L=200$ : we expect the moments of the observables to converge towards their asymptotic limits (corresponding to infinite tessellations) when $L \rho \gg 1$ so that finitesize effects fade away. The number of realizations is $5 \times 10^{3}$ for each size $L$.

The simulation results for the average volume $\left\langle V_{3}\right\rangle$, the second moment $\left\langle V_{3}^{2}\right\rangle$, the average surface $\left\langle S_{3}\right\rangle$ and the correlations $\left\langle V_{3} S_{3}\right\rangle$ between the volume and the surface are given in Tabs. 2, 5, respectively, for $L=200$. Numerical findings are in good agreement with the theoretical formulas, and the minimum of all these quantities is attained for the case of isotropic tessellations, as predicted by the theory. The evolution of these moments as a function of the side $L$ of the box is shown in Figs. 3 and 4 . for small $L$, finite-size effects are clearly visible; for larger $L$, the estimated moments converge to their asymptotic limits for infinite tessellations. The rate of convergence to the asymptotic values depends on the anisotropy law $H(\mathbf{n})$ : the stronger the anisotropy, the slower the convergence. Isotropic and box tessellations, although converging to different limits, appear to have the nearly same rate of convergence, which pos- 

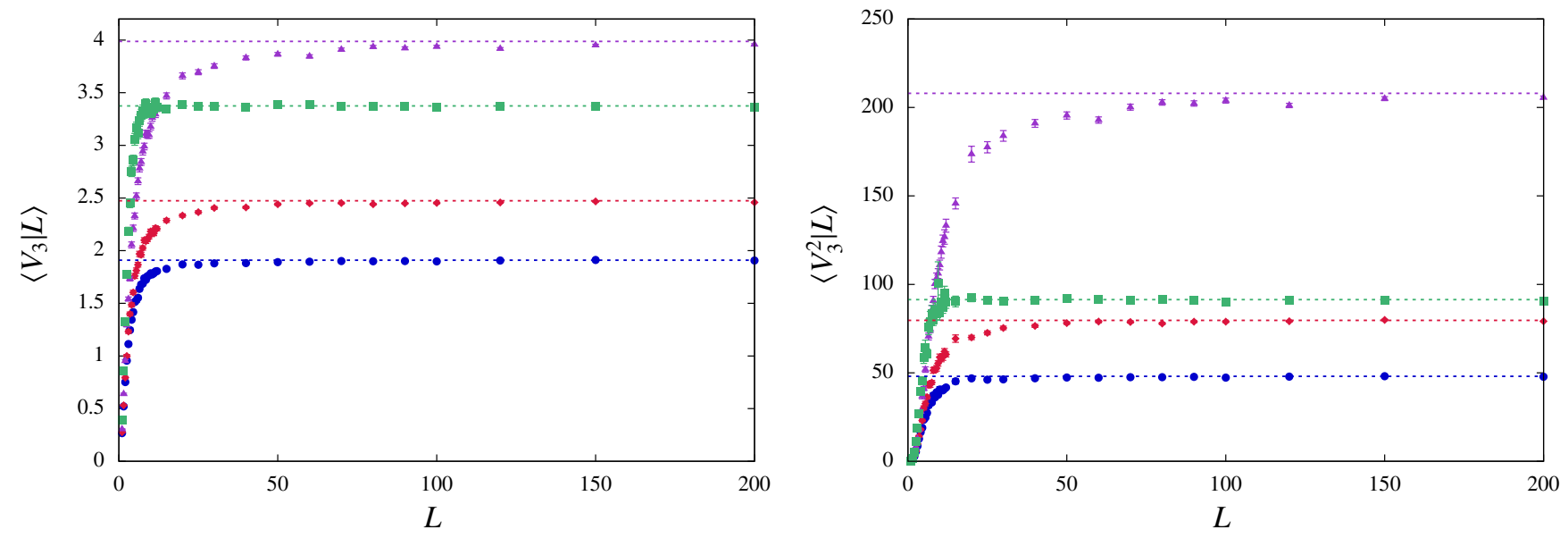

Figure 3: Average volume $\left\langle V_{3}\right\rangle$ (top) and second moment of the volume $\left\langle V_{3}^{2}\right\rangle$ (bottom) of Poisson tessellations restricted to a box of side $L$, with various anisotropy laws $H(\mathbf{n})$, for $d=3$. The tessellation density is $\rho=1$ for all the angular laws $H(\mathbf{n})$. Symbols denote Monte Carlo simulation results: blue circles correspond to isotropic distribution, red diamonds to quadratic distribution, green squares to box distribution and purple triangles to histogram distribution. Solid lines correspond to the respective limits for infinite Poisson tessellations, as given in Eqs. (7) and (11), respectively.

\begin{tabular}{ccc}
\hline \hline Law $H(\mathbf{n})$ & $\left\langle V_{3} S_{3}\right\rangle$ & Monte Carlo \\
\hline Isotropic & 96 & $95.7 \pm 0.3$ \\
Quadratic & $\simeq 159.2455$ & $158.6 \pm 0.6$ \\
Box & $729 / 4=182.25$ & $181.2 \pm 0.7$ \\
Histogram & $\simeq 415.9862$ & $411 \pm 2$ \\
\hline \hline
\end{tabular}

Table 5: Volume-surface correlations $\left\langle V_{3} S_{3}\right\rangle$ of Poisson tessellations restricted to a box of side $L=200$, with various anisotropy laws $H(\mathbf{n})$, for $d=3$. The tessellation density is $\rho=1$ for all the angular laws $H(\mathbf{n})$. The limit value for infinite Poisson tessellations is given in Eq. 20.

\begin{tabular}{ccc}
\hline \hline Law $H(\mathbf{n})$ & $\left\langle C_{3}\right\rangle$ & Monte Carlo \\
\hline Isotropic & 6 & $6 \pm 7 \times 10^{-8}$ \\
Quadratic & 6 & $6 \pm 9 \times 10^{-8}$ \\
Box & 6 & $6 \pm 0$ \\
Histogram & 6 & $6 \pm 10^{-7}$ \\
\hline \hline
\end{tabular}

Table 6: Average number of faces $\left\langle C_{3}\right\rangle$ in Poisson tessellations restricted to a box of side $L=200$, with various anisotropy laws $H(\mathbf{n})$, for $d=3$. The tessellation density is $\rho=1$ for all the angular laws $H(\mathbf{n})$. The limit value for infinite Poisson tessellations is $\left\langle C_{3}\right\rangle=6$ for any $H(\mathbf{n})$.

sibly stems from box tessellations being quasi-isotropic. Inspection of Fig. 3 shows that $\left\langle V_{3}\right\rangle$ and $\left\langle V_{3}^{2}\right\rangle$ appear to have similar rates of convergence, for a given $H(\mathbf{n})$.

The Monte Carlo simulation results for the average number ${ }_{265}$ of faces $\left\langle C_{3}\right\rangle$ of each cell are given in Tab. 6 for $L=200$ : it is immediately apparent that $\left\langle C_{3}\right\rangle$ does not depend on the angular law $H(\mathbf{n})$, as expected. The distribution of the number of faces $C_{3}$ has been also estimated by Monte Carlo simulation and is displayed in Fig. 5 for $L=200$ and $\rho=1$. Intriguingly, the curves corresponding to different laws $H(\mathbf{n})$ almost collapse onto the same functional form, apart from the case of 270 Box tessellations, for which a distinct behaviour is observed: by construction, the number of faces in Box tessellations is exactly equal to 6 for each cell.

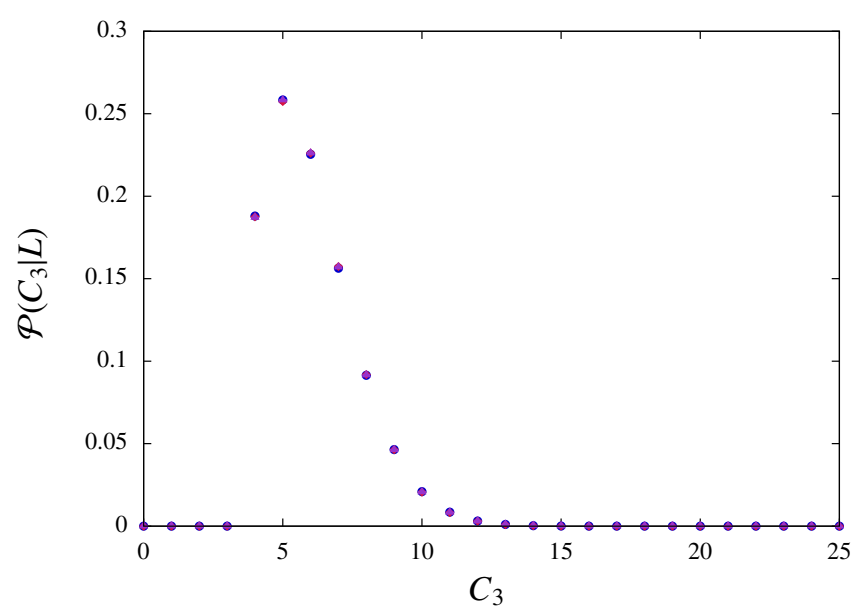

Figure 5: Distribution of the number of faces $C_{3}$ in Poisson tessellations cells restricted to a box of side $L=200$, with various anisotropy laws $H(\mathbf{n})$, for $d=3$. The tessellation density is $\rho=1$ for all the angular laws $H(\mathbf{n})$. Symbols denote Monte Carlo simulation results: blue circles correspond to isotropic distribution, red diamonds to quadratic distribution and purple triangles to histogram distribution. For Box tessellations, $C_{3}$ is trivially equal to 6 for each cell.

Simulation details concerning the inradius and the outradius are given in Appendix C, for the sake of completeness.

\subsection{Chord lengths}

As a first verification test, we have estimated the chord length distribution $f(\ell \mid \boldsymbol{\Omega}, L)$ corresponding to lines having a fixed orientation $\Omega$ within a box of side $L$, for several anisotropy laws $H(\mathbf{n})$. Monte Carlo simulation results are shown in Fig. 6 for $L=150$ and $\rho=1$. An ensemble of $5 \times 10^{3}$ realizations and $10^{3}$ sample lines for each geometry have been taken. Furthermore, Monte Carlo simulation results for the chord length distribution 

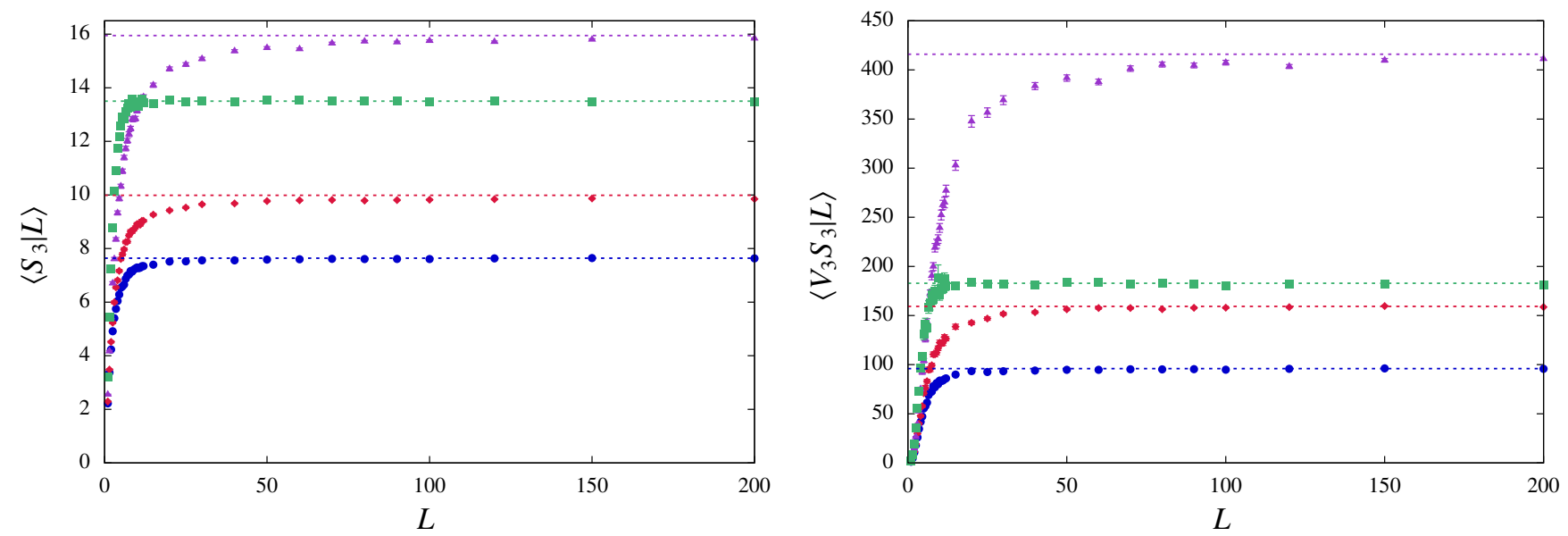

Figure 4: Average surface $\left\langle S_{3}\right\rangle$ (top) and volume-surface correlations $\left\langle V_{3} S_{3}\right\rangle$ (bottom) of Poisson tessellations restricted to a box of side $L$, with various anisotropy laws $H(\mathbf{n})$, for $d=3$. The tessellation density is $\rho=1$ for all the angular laws $H(\mathbf{n})$. Symbols denote Monte Carlo simulation results: blue circles correspond to isotropic distribution, red diamonds to quadratic distribution, green squares to box distribution and purple triangles to histogram distribution. Solid lines correspond to the respective limits for infinite Poisson tessellations, as given in Eqs. [19] and 20], respectively.

\begin{tabular}{ccc}
\hline \hline$H(\mathbf{n})$ & $\Lambda_{c}$ & Monte Carlo \\
\hline Isotropic & 1 & $0.9940 \pm 8 \times 10^{-4}$ \\
Quadratic & 1 & $0.9937 \pm 8 \times 10^{-4}$ \\
Box & 1 & $0.9927 \pm 8 \times 10^{-4}$ \\
Histogram & 1 & $0.9937 \pm 8 \times 10^{-4}$ \\
\hline \hline
\end{tabular}

Table 7: Average correlation length $\Lambda_{c}$ for Poisson tessellations restricted to a box of side $L=150$, with various anisotropy laws $H(\mathbf{n})$, with $d=3$. The tessellation density is $\rho=1$ for all the angular laws $H(\mathbf{n})$. The line orientation is uniform and isotropic. The line orientation is uniform and isotropic. The limit value for infinite Poisson tessellations is $\Lambda_{c}=1 / \rho$, as in Eq. [39].

\begin{tabular}{ccc}
\hline \hline$H(\mathbf{n})$ & $\Lambda_{c}^{4}$ & Monte Carlo \\
\hline Isotropic & 24 & $23.40 \pm 0.08$ \\
Quadratic & 30.71 & $30.0 \pm 0.1$ \\
Box & 25.78 & $25.0 \pm 0.1$ \\
Histogram & 49.79 & $48.1 \pm 0.2$ \\
\hline \hline
\end{tabular}

Table 8: The fourth moment $\Lambda_{c}^{4}$ of the chord length for Poisson tessellations restricted to a box of side $L=150$, with various anisotropy laws $H(\mathbf{n})$, with $d=3$. The tessellation density is $\rho=1$ for all the angular laws $H(\mathbf{n})$. The line orientation is uniform and isotropic. The limit value for infinite Poisson 300 tessellations is $\Lambda_{c}^{4}=\eta_{3} \Gamma(5) / \rho^{4}$, as in Eq. 48.

$f_{\alpha}(\ell \mid \boldsymbol{\Omega}, L)$ through material $\alpha$ corresponding to lines having a fixed orientation $\boldsymbol{\Omega}$ are displayed in Fig. 7 for various values of the color probability $p_{\alpha}$. An ensemble of $5 \times 10^{3}$ realizations and $10^{2}$ sample lines for each geometry have been taken. Numerical findings for both $f(\ell \mid \mathbf{\Omega}, L)$ and $f_{\alpha}(\ell \mid \mathbf{\Omega}, L)$ are in good agreement with the exponential densities given in Eqs. 26) and (31), respectively, which are exact for infinite tessellations. Finite-size effects are negligible, since $L \rho \gg 1$ for these simulations.

The special case of chord lengths induced by lines that are homogeneously and isotropically distributed has been separately considered. Simulation results for the resulting average trated in Tab. 7. where Monte Carlo estimates are compared to the expected theoretical value $\Lambda_{c}=1 / \rho$. For such a large $L$, finite-size effects are rather weak and the simulation findings agree with the limit values. The behaviour of the average correlation length $\Lambda_{c}(L)$ as a function of the system size $L$ is shown in Fig. 8 (left). Numerical findings confirm that $\Lambda_{c}(L)$ does not depend on the anisotropy law $H(\mathbf{n})$. Moreover, the convergence is not affected by the anisotropy law $H(\mathbf{n})$, either.

For the purpose of comparison, we have also investigated the behaviour of the chord length estimator

$$
\Lambda_{c}^{*} \equiv \mathbb{E}\left[\frac{\sum_{j=1}^{q} \ell_{j}}{q}\right],
$$

which is related to $\int_{\Omega_{d}^{+}} \Lambda(\boldsymbol{\Omega}) d U(\boldsymbol{\Omega})=\eta_{1} / \rho$. The corresponding Fig. 8 (right). Generally speaking, we observe that $\Lambda_{c}^{*} \geq \Lambda_{c}=$ $1 / \rho$, as expected: this is particularly apparent for the quadratic distribution and for the histogram distribution. These numerical findings are consistent with the theoretical analysis provided by Miles (34). Moreover, the Box distribution yields results that are almost superposed to those of the isotropic distribution.

Finally, we have considered the fourth moment $\Lambda_{c}^{4}$ of the chord lengths for Poisson tessellations restricted to a box of side $L=150$. In Tab. 8 the simulation results corresponding to various anisotropy laws $H(\mathbf{n})$ are compared to the limit value $\Lambda_{c}^{4}=\eta_{3} \Gamma(5) / \rho^{4}$, as given in Eq. (48). The agreement is satisfactory, although finite-size effects are apparent because of the large powers of the lengths. The Box distribution yields again results that are close to those of the isotropic distribution. The behaviour of the average correlation length $\Lambda_{c}(L)$ as a function of the system size $L$ is shown in Fig. 9, where we compare the estimator for $\Lambda_{c}^{d+1}$ to

$$
\Lambda_{c}^{d+1, *} \equiv \mathbb{E}\left[\frac{\sum_{j=1}^{q} \ell_{j}^{d+1}}{q}\right],
$$



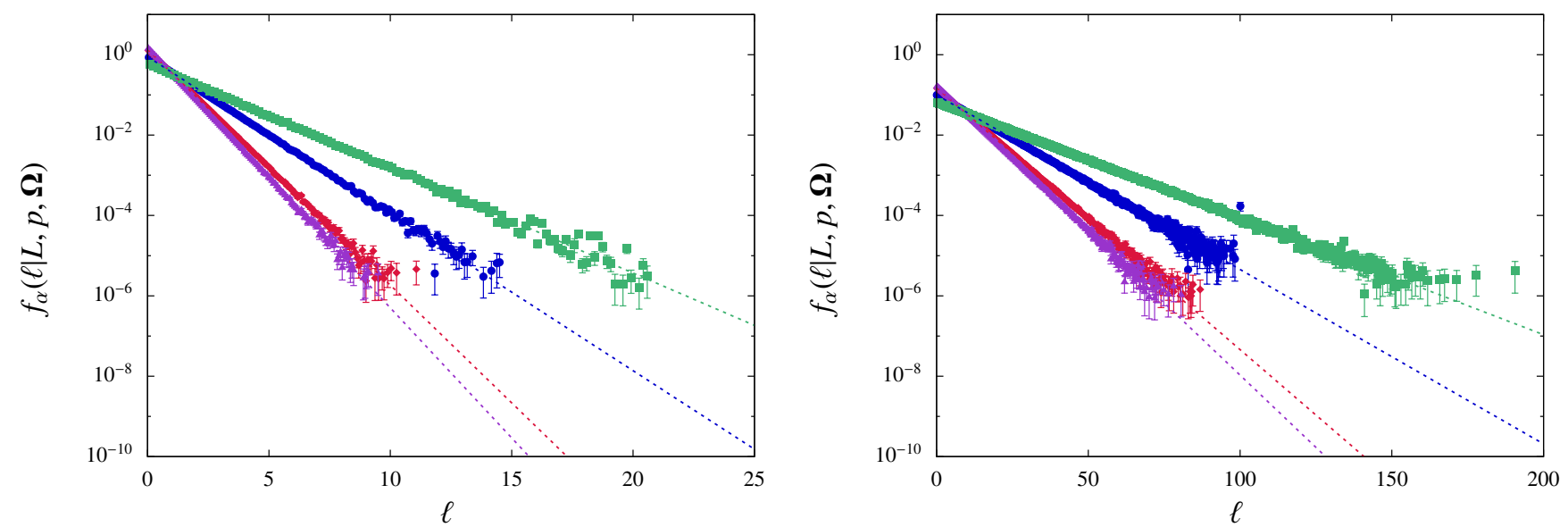

Figure 7: Chord length distribution $f_{\alpha}(\ell \mid L, \Omega)$ through clusters of material $\alpha$ in Poisson tessellations restricted to a box of side $L=150$, with various anisotropy laws $H(\mathbf{n})$, for $d=3$, with $p_{\alpha}=0.1$ (left) and $p_{\alpha}=0.9$ (right). The tessellation density is $\rho=1$ for all the angular laws $H$ (n). The line orientation is chosen as $\theta=0$. Symbols denote Monte Carlo simulation results: blue circles correspond to isotropic distribution, red diamonds to quadratic distribution, green squares to box distribution and purple triangles to histogram distribution. The solid lines correspond to the limit exponential density given in Eq. 31, with average $\Lambda_{\alpha}(\boldsymbol{\Omega})=\Lambda(\boldsymbol{\Omega}) /\left(1-p_{\alpha}\right)$.

which is related to $\int_{\Omega_{d}^{+}} \Lambda^{d+1}(\mathbf{\Omega}) d U(\mathbf{\Omega})=\eta_{d+1} \Gamma(d+2) / \rho^{d+1}$. Numerical investigations show that $\Lambda_{c}^{4} \leq \Lambda_{c}^{4, *}$, as expected.

\section{Conclusions}

Markov media provide a prototype model for linear particle transport in random materials, although their practical realizability in arbitrary dimension has been a long-standing open ${ }^{340}$ question. In this work we have shown that Poisson hyperplane tessellations allow for an explicit construction of random media sarkov property and easily including anisotropy. The chord length distribution of Poisson hyperplane tessellations is exactly exponential (in the limit case of tessellations having an infinite extension), with a direction-dependent average that is a function of the anisotropy law $H(\mathbf{n})$.

We have shown that for uniform and isotropic lines the average chord length and the $(d+1)$-moment are intimately related to the statistical features of the tessellation cells, such as the surface-to-volume ratio, and can be expressed by elegant formulas. In particular, the average chord length is insensitive to the anisotropy law $H(\mathbf{n})$, whereas the $(d+1)$-moment does depend on $H(\mathbf{n})$.

In order to investigate the properties of anisotropic Markov media we have developed a computer code to generate anisotropic Poisson tessellations in arbitrary dimension restricted to a finite box of side $L$. The required algorithms have been obtained by generalizing our previous work concerning isotropic tessellations. We have carefully analyzed the convergence to exact asymptotic formulas for the chord length distribution and the polyhedral features of the tessellation cell by extensively using Monte Carlo simulation. The impact of anisotropy has been illustrated by resorting to a few relevant examples of angular distributions in dimension $d=3$. In the limit of very large $L$ we have shown that finite-size effects fade away and the properties of the tessellations converge to the theoretical results.

The results presented in this paper may encourage further theoretical work concerning the properties of Poisson hyperplane tessellations and might stimulate similar analyses for other classes of random media.

\section{Acknowledgements}

C. Larmier and A. Zoia wish to thank Électricité de France (EDF) for partial financial support. The authors express their gratitude to F. Malvagi and A. Mazzolo (CEA) and to E. Dumonteil (IRSN) for many fruitful discussions.

\section{Appendix A. Inequalities for anisotropic tessellations}

The volume $V_{d}$ and the surface $S_{d}$ depend on the anisotropy law $H(\mathbf{n})$. For any sufficiently well-behaved $H(\mathbf{n})$, we have

$$
\zeta_{d} \leq \zeta_{d}^{\text {iso }}
$$

which from Eq. (7) implies that the average volume attains a minimum for the case of isotropic Poisson tessellations (45, 38), namely,

$$
\left\langle V_{d}\right\rangle \geq\left\langle V_{d}\right\rangle^{\text {iso }} .
$$

More generally, it can be proven that $\left\langle V_{d}^{m}\right\rangle \geq\left\langle V_{d}^{m}\right\rangle^{\text {iso }}$ for the moments of any order $m \geq 1$ (45), whence in particular

$$
\left\langle V_{d}^{2}\right\rangle \geq\left\langle V_{d}^{2}\right\rangle^{\text {iso }} \text {. }
$$

Moreover, it has been shown that $\mathrm{cv}^{\text {box }} \leq \mathrm{cv} \leq \mathrm{cv}^{\text {iso }}$ (38).

For $S_{d}$, Eq. (19) implies that the average surface attains also a minimum for the case of isotropic Poisson tessellations, namely,

$$
\left\langle S_{d}\right\rangle \geq\left\langle S_{d}\right\rangle^{\text {iso }}
$$



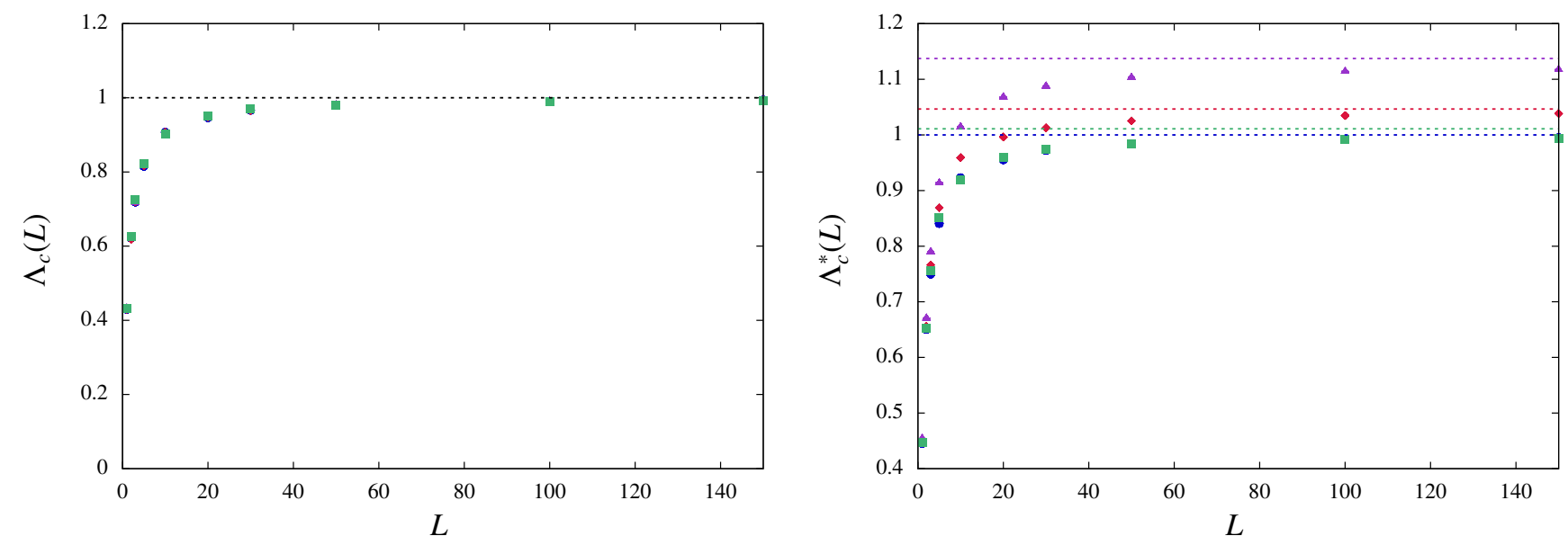

Figure 8: The average correlation length $\Lambda_{c}$ (left) and the estimator $\Lambda_{c}^{*}$ (right) in Poisson tessellation cells for a box of side $L$, as a function of $L$, with various anisotropy laws $H(\mathbf{n})$, for $d=3$. The tessellation density is $\rho=1$ for all the angular laws $H(\mathbf{n})$. The line orientation is uniform and isotropic. Symbols denote Monte Carlo simulation results: blue circles correspond to isotropic distribution, red diamonds to quadratic distribution, green squares to box distribution and purple triangles to histogram distribution. The limit values for infinite Poisson tessellations (dashed lines) are $\Lambda_{c}=1 / \rho$, as in Eq. 39], and $\Lambda_{c}^{*}=\eta_{1} / \rho$.

From Eqs. (A.3) and (20), we have an analogous inequality for the correlations, namely,

$$
\left\langle V_{d} S_{d}\right\rangle \geq\left\langle V_{d} S_{d}\right\rangle^{\text {iso }} .
$$

\section{Appendix B. Cauchy formulas}

In dimension $d=2$, a line section $\mathcal{T}$ of a bounded domain $X \subset \mathbb{R}^{2}$ ensuring homogeneity and isotropy can be obtained as follows. Consider the disk $S$ containing $X$ and with the smallest ${ }_{375}$ possible radius. Take then an isotropic direction $\boldsymbol{\Omega}$ emanating from the center of $S$. Take a random point whose position $z$ is uniformly distributed on the diameter of $S$ in direction $\boldsymbol{\Omega}$. Construct now the line passing through $z$ and orthogonal to $\boldsymbol{\Omega}$. If the line intersects the domain $X$, this is an acceptable line section satisfying the properties above; if not, repeat the whole procedure until a new line hits $X$.

In dimension $d=3$, a line section $\mathcal{T}$ of a bounded domain $X \subset \mathbb{R}^{3}$ satisfying the homogeneity and isotropy property can be obtained by first constructing a plane section. Consider the sphere $S$ containing $X$ and with the smallest possible radius. Take then an isotropic direction $\boldsymbol{\Omega}$ emanating from the center of $S$. Take a random point whose position $z$ is uniformly distributed on the diameter of $S$ in direction $\boldsymbol{\Omega}$. Construct now the plane passing through $z$ and orthogonal to $\boldsymbol{\Omega}$. If the plane intersects the domain $X$, this is a plane section of $X$ satisfying homogeneity and isotropy; if not, repeat the whole procedure until a new plane hits $X$. Once the plane section is obtained, the procedure for line sections in $d=2$ can be applied: it can be shown that an homogeneous and isotropic line section of an homogeneous and isotropic plane section of $X$ is actually an homogeneous and isotropic line section of $X$ in $d=3$.

Other procedures exist for homogeneous and isotropic line sections in dimension $d$ that do not require rejection methods (48). This can be achieved, e.g., by first choosing a point
P uniformly on the surface of $X \subset \mathbb{R}^{d}$ and then sampling lines passing through $\mathbf{P}$ and with direction $\boldsymbol{\Omega}$ satisfying an isotropic incident flux with respect to the normal $\mathbf{n}$ entering the surface of $X$ at point $\mathbf{P}$. This condition imposes the measure (18, 48)

$$
\boldsymbol{\Omega} \cdot \mathbf{n} \frac{d \boldsymbol{\Omega}}{\kappa_{d-1}} \frac{d S_{d}(X)}{S_{d}(X)} .
$$

The term $\cos \theta=\boldsymbol{\Omega} \cdot \mathbf{n}$ implies that in polar coordinates trajectories starting on the surface must enter the domain $X$ with $\theta=\arcsin (2 s-1)$ in two dimensions and $\theta=1 / 2 \arccos (1-2 s)$ in three dimensions, $s$ being uniformly distributed in $(0,1]$ (49).

Consider then a bounded domain $X$ in $\mathbb{R}^{d}$ and an ensemble of homogeneous and isotropic random lines $\mathcal{T}$. The probability that a line drawn from this ensemble hits a sub-domain $Y \subset X$ is given by

$$
P(\mathcal{T} \cap Y \neq 0)=\frac{S_{d}(Y)}{S_{d}(X)},
$$

where $S_{d}(A)$ denotes the surface of a domain $A$ in $\mathbb{R}^{d}(46)$. Furthermore, conditionally to hitting $Y$, such lines are homogeneous and isotropic through $Y$. This result is independent of the position and orientation of the sub-domain $Y$ within $X$. Let us denote by $\ell$ the random line sections induced on $Y$. The Cauchy formula (also called Crofton second theorem) states that the ensemble average $\mathbb{E}[\ell]$ of the random line sections through $Y$ satisfies

$$
\mathbb{E}[\ell]=\alpha_{d} \frac{V_{d}(Y)}{S_{d}(Y)},
$$

where $V_{d}(A)$ denotes the volume of a domain $A$ in $\mathbb{R}^{d}$, provided that lines are homogeneous and isotropic (46, 34). In other words, the average line section $\mathbb{E}[\ell]$ through $Y$ depends only on the ratio between the volume and the surface of $Y$, regardless of the specific details of the shape of the domain, up to the dimension-dependent constant $\alpha_{d}$. This is a special case of a broader set of stereological formulas derived by Miles for the 

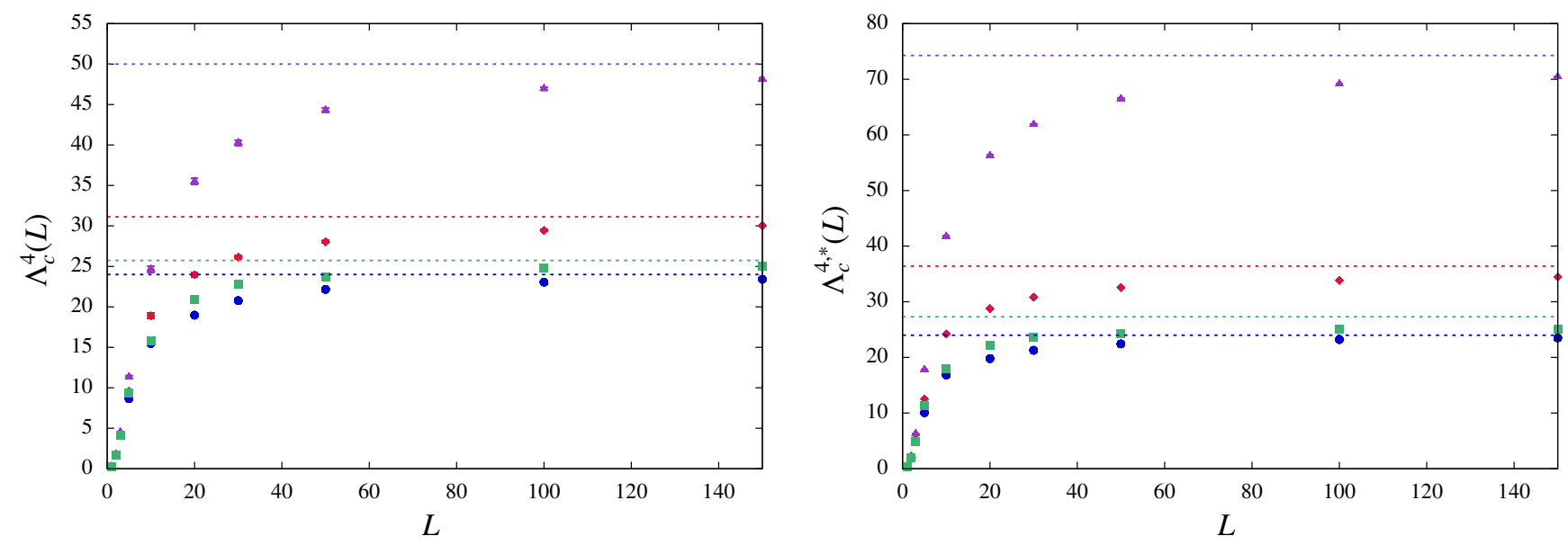

Figure 9: The 4-th moment $\Lambda_{c}^{4}$ (left) and the estimator $\Lambda_{c}^{4, *}$ (right) in Poisson tessellation cells for a box of side $L$, as a function of $L$, with various anisotropy laws $H(\mathbf{n})$, for $d=3$. The tessellation density is $\rho=1$ for all the angular laws $H(\mathbf{n})$. The line orientation is uniform and isotropic. Symbols denote Monte Carlo simulation results: blue circles correspond to isotropic distribution, red diamonds to quadratic distribution, green squares to box distribution and purple triangles to histogram distribution. The limit values for infinite Poisson tessellations (dashed lines) are $\Lambda_{c}^{4}=\eta_{3} \Gamma(5) / \rho^{4}$, as in Eq. 48 , and $\Lambda_{c}^{4, *}=\eta_{4} \Gamma(5) / \rho^{4}$

projection of a body (or a collection of bodies) onto random

[2] Murata I, Mori T, Nakagawa M. Continuous energy Monte Carlo calculations of randomly distributed spherical fuels in High-Temperature GasCooled Reactors based on a statistical geometry model. Nucl Sci Eng 1996;123:96-109.

Another remarkable consequence of the Crofton theorem is that the $(d+1)$-th moment $\mathbb{E}\left[\ell^{d+1}\right]$ of the random line sections through $Y$ satisfies $(34)$

$$
\mathbb{E}\left[\ell^{d+1}\right]=\gamma_{d} \frac{V_{d}^{2}(Y)}{S_{d}(Y)},
$$

where the constant $\gamma_{d}$ is given in Eq. (45).

\section{Appendix C. Analysis of the inradius and the outradius}

The distribution of the inradius $r_{\mathrm{in}, 3}$ has been also estimated by Monte Carlo simulation and is provided in Fig. C.10 for large $L=200$ and $\rho=1$. It is immediately apparent that the curves corresponding to different laws $H(\mathbf{n})$ collapse onto $_{420}$ the same functional form, which for infinite tessellations is the exponential distribution

$$
f\left(r_{\mathrm{in}, 3}\right)=4 \rho e^{-4 \rho r_{\mathrm{in}, 3}},
$$

where we have used $\alpha_{3}=4$. In particular, the average inradius yields $\left\langle r_{\mathrm{in}, 3}\right\rangle=1 /(4 \rho)$ for infinite tessellations. Finite-size effects are negligible, since $\rho L \gg 1$. As mentioned above, to the ${ }_{430}$ best of our knowledge the theoretical behaviour of the outradius for infinite tessellations is not known (not even for the simplest case of isotropic tessellations). Monte Carlo simulations show however that $\left\langle r_{\text {out }, 3}\right\rangle$ non-trivially depends on the law $H(\mathbf{n})$, con- $_{435}$ trary to the inradius.

\section{References}

[1] Pomraning GC. Linear kinetic theory and particle transport in stochastic mixtures. River Edge, NJ, USA: World Scientific Publishing; 1991.

[3] Wu Q, Kim S, Ishii M, Beus S. One-group interfacial area transport in vertical bubbly flow. Int J Heat Mass Transf 1998:41;1103-12.

[4] Levermore CD, Pomraning GC, Sanzo DL, Wong J. Linear transport theory in a random medium. J Math Phys 1986;27:2526-36.

[5] Zimmerman GB, Adams ML. Algorithms for Monte Carlo particle transport in binary statistical mixtures. Trans Am Nucl Soc 1991:66;287.

[6] Levermore CD, Zimmerman GB. Modeling charged particle loss in a fuel/shell mixture. J Math Phys 1993:34;4725-29.

[7] Haran O, Shvarts D, Thieberger R. Transport in 2D scattering stochastic media: simulations and models. Phys Rev E 2000:61;6183-89.

[8] Williams MMR. Ann Nucl Energy Uncertainties in control rod worth in a damaged reactor core. Ann Nucl Energy 2004:31;1073-81.

[9] Larmier C, Zoia A, Malvagi F, Dumonteil E, Mazzolo A, Neutron multiplication in random media: reactivity and kinetics parameters. Ann Nucl Energy 2018:111;391-406.

[10] Doub WB. Particle self-shielding in plates loaded with spherical poison particles. Nucl Sci Eng 1961:10;299-307.

[11] Yamamoto T. Study on double heterogeneity effect of Pu-rich agglomerates in mixed oxide fuel using cross section homogenization method for particle-dispersed media. Ann Nucl Energy 2010:37;398-405.

[12] Davis AB, Marshak A. Photon propagation in heterogeneous optical media with spatial correlations. J Quant Spectrosc Radiat Transfer 2004:84;3-34.

[13] Kostinski AB, Shaw RA. Scale-dependent droplet clustering in turbulent clouds. J Fluid Mech 2001:434;389-98.

[14] Malvagi F, Byrne RN, Pomraning GC, Somerville RCJ. Stochastic radiative transfer in partially cloudy atmosphere. J Atm Sci 1992:50;2146-58.

[15] Kassianov E, Veron DE. Stochastic radiative transfer in Markovian mixtures: Past, present, and future. J Quant Spectrosc Radiat Transfer 2011:112;566-76.

[16] Tuchin V. Tissue optics: light scattering methods and instruments for medical diagnosis. Cardiff, UK: SPIE Press; 2007.

[17] Sanchez R. Linear kinetic theory in stochastic media. J Math Phys 1988;30:2498-2511.

[18] Santaló LA. Integral geometry and geometric probability. Reading, MA, USA: Addison-Wesley; 1976.

[19] Zuchuat O, Sanchez R, Zmijarevic I, Malvagi F. Transport in renewal statistical media: benchmarking and comparison with models. J Quant Spectrosc Radiat Transfer 1994;51:689-722.

[20] Larmier C, Zoia A, Malvagi F, Dumonteil E, Mazzolo A. Monte Carlo 


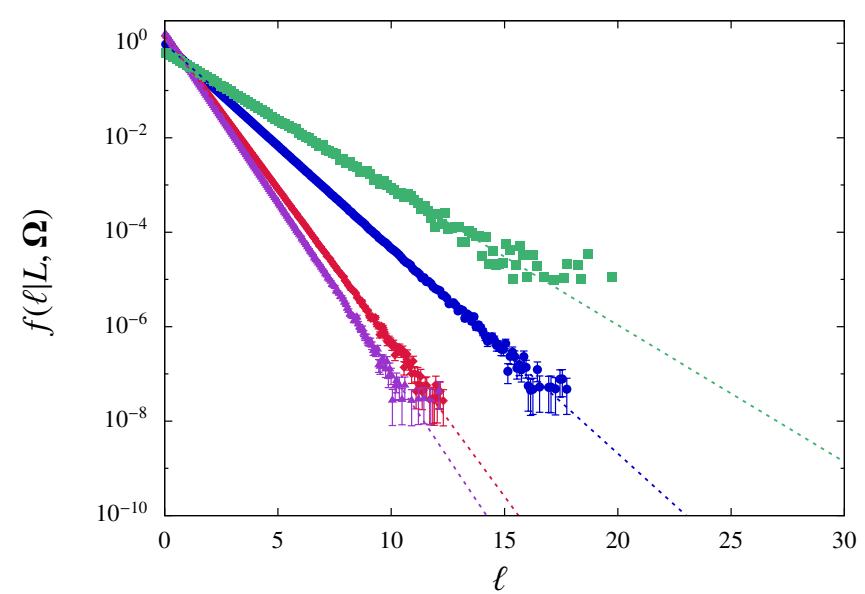

Figure 6: Chord length distribution $f(\ell \mid L, \mathbf{\Omega})$ in Poisson tessellations restricted to a box of side $L=150$, with various anisotropy laws $H(\mathbf{n})$, for $d=3$. The tessellation density is $\rho=1$ for all the angular laws $H(\mathbf{n})$. The line orientation is chosen as $\phi=\theta=\pi / 4$. Symbols denote Monte Carlo simulation results: blue circles correspond to isotropic distribution, red diamonds to quadratic distribution, green squares to box distribution and purple triangles to histogram distribution. The solid lines correspond to the limit exponential density given in Eq. (26), with average $\Lambda(\boldsymbol{\Omega})$ depending on the orientation $\boldsymbol{\Omega}$ and on the law $H(\mathbf{n})$ (see Eq. 27) ). For the isotropic distribution we have $\Lambda(\boldsymbol{\Omega})=1$, for the quadratic distribution $\Lambda(\boldsymbol{\Omega})=2 / 3$, for the box distribution $\Lambda(\boldsymbol{\Omega})=3 / 2$, and for the histogram distribution $\Lambda(\boldsymbol{\Omega}) \simeq 0.60383$.

particle transport in random media: The effects of mixing statistics. J Quant Spectrosc Radiat Transfer 2017:196;270-86.

[21] Pomraning GC. Statistics, renewal theory and particle transport. J Quant ${ }^{485}$ Spectrosc Radiat Transfer 1989;42:279-93.

[22] Levermore CD, Pomraning GC, Wong J. Renewal theory for transport processes in binary statistical mixtures. J Math Phys 1988;29:995-1004.

[23] Dixmier M. Une nouvelle description des empilements aléatoires et des ${ }_{490}$ fluides denses. J Phys France 1978; 39:873-95.

[24] Lu B, Torquato S, Lineal-path function for random heterogeneous materials. Phys Rev A 1992;45:992.

[25] Torquato S, Lu B. Chord-length distribution function for two-phase random media. Phys Rev E 1993;47:2950.

[26] Mazzolo A, Roesslinger B. Monte Carlo simulation of the chord length ${ }^{495}$ distribution function across convex bodies, non-convex bodies and random media. Monte Carlo Meth Appl 2004;10:443-54.

[27] Guéron D, Mazzolo A. Properties of chord length distributions across ordered and disordered packing of hard spheres. Phys Rev E 2003; 68:066117.

[28] Olson GL, Miller DS, Larsen EW, Morel JE. Chord length distributions in binary stochastic media in two and three dimensions. J Quant Spectrosc Radiat Transfer 2006:101;269-83.

[29] Donovan TJ, Danon Y. Application of Monte Carlo chord-length sampling algorithms to transport through a two-dimensional binary stochastic ${ }^{505}$ mixture. Nucl Sci Eng 2003;143:226-39.

[30] Torquato S. Random heterogeneous materials: microstructure and macroscopic properties. New York, USA: Springer-Verlag; 2002.

[31] Lepage T, Delaby L, Malvagi F, Mazzolo A. Monte Carlo simulation of fully Markovian stochastic geometries. Prog Nucl Sci Techn 2011:2;74348.

[32] Larmier C, Dumonteil E, Malvagi F, Mazzolo A, Zoia A. Finite-size effects and percolation properties of Poisson geometries. Phys Rev E 2016:94;012130.

[33] Miles RE. The random division of space. Suppl Adv Appl Prob 1972:4;243-66.

[34] Miles RE. Spatial tessellations and their stereology. In: Science on form. Proceedings of the first international symposium for science on form, S. Ishizaka general editor, 147-155. Tokyo, Japan: KTK Scientific Pub-

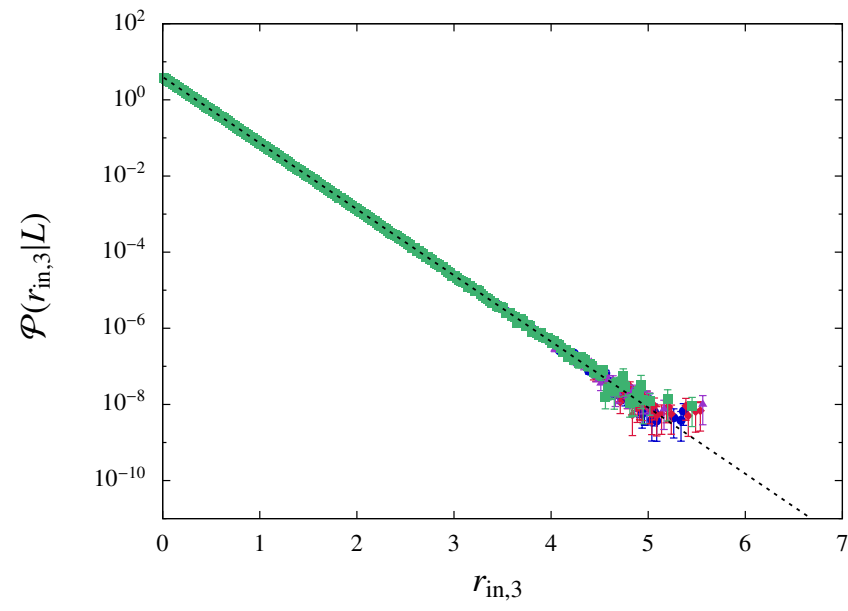

Figure C.10: Distribution of the in-radius $r_{\mathrm{in}, 3}$ of the Poisson tessellation cells for a box with side $L=200$, with various anisotropy laws $H(\mathbf{n})$, for $d=3$. The tessellation density is $\rho=1$ for all the angular laws $H(\mathbf{n})$. Symbols denote Monte Carlo simulation results: blue circles correspond to isotropic distribution, red diamonds to quadratic distribution, green squares to box distribution and purple triangles to histogram distribution. The solid line corresponds to the limit distribution for infinite Poisson tessellations, i.e., the exponential density given in Eq. [C.1], independent of $H(\mathbf{n})$.

lisher; 1986.

[35] Pomraning GC. Diffusive transport in binary anisotropic stochastic mixtures. Ann Nucl Energy 1992:19;737-63.

[36] Marinosci A, Larmier C, Zoia A, Neutron transport in anisotropic random media. Ann Nucl Energy 2018:118;406-13.

[37] Miles RE. A synopsis of Poisson flats in Euclidean spaces. Izv Akad Nauk Arm SSR Ser Mat 1970:5;263-85.

[38] Schneider R, Weil W. Stochastic and integral geometry. Berlin, Germany: Springer; 2008.

[39] Serra J. Image analysis and mathematical morphology. London, UK: Academic Press; 1982.

[40] Ambos AYu, Mikhailov GA. Statistical simulation of an exponentially correlated many-dimensional random field. Russ J Numer Anal Math Modelling 2011:26;263-73.

[41] Miles RE. Random polygons determined by random lines in a plane. Proc Nat Acad Sci USA 1964: 52; 901-7.

[42] Goudsmit S. Random distribution of lines in a plane. Rev Mod Phys 1945:17;321.

[43] Schneider R. Extremal properties of random mosaics. In: Geometry - Intuitive, Discrete, and Convex. A Tribute to Làszlò Fejes Tòth (I. Bàràny, K. J. Böröczky, G. Fejes Tòth, J. Pach, eds), Springer, Berlin. Bolyai Soc Math Studies 2013:24;301-30.

[44] Schneider R. Second moments related to Poisson hyperplane tessellations. J Math Anal Appl 2016:434;1365-75.

[45] Mecke J. Inequalities for the anisotropic poisson polytope. Adv Appl Prob 1995:27;56-62.

[46] Miles RE. Multi-dimensional perspectives on stereology. J Microscopy 1972:95; 181-95.

[47] Miles RE. A comprehensive set of stereological formulae for embedded aggregates of not necessarily convex particles. J Microscopy 1985:138;115-25.

[48] Coleman R. Random paths through convex bodies. J Appl Probab 1969:6;430-41.

[49] Zoia A, Dumonteil E, Mazzolo A, Properties of branching exponential flights in bounded domains. EPL 2012:100;40002. 